\title{
Técnica Lecretexor para dinamizar la enseñanza y aprendizaje de la ortografía
}

\section{Lecretexor technique to stimulate the teaching and learning of spelling}

\author{
Niel Agripino Palomino Gonzales ${ }^{1}$ \\ ${ }^{1}$ Universidad Nacional de San Antonio Abad del Cusco. Av de la Cultura No 733, Cuzco, Perú. Correo \\ electrónico: niel.palomino@unsaac.edu.pe. ORCID: https://orcid.org/0000-0002-3607-4939
}

\section{Resumen}

El objetivo de la investigación es la determinación de la forma en que la aplicación de la técnica LECRETEXOR (lectura y creación de textos ortográficos) influye en el aprendizaje de la ortografía. La metodología empleada fue la cuasiexperimental. Los sujetos de la muestra constituyeron las alumnas del primer grado de secundaria (Primero A, Grupo Experimental) y (Primero B, Grupo Control) de la I. E. P. de Señoritas "Juan Landázuri Ricketts" - Cusco. Las principales conclusiones a que se arribaron son: que aplicando la técnica LECRETEXOR en varias sesiones de aprendizaje, se logra que las alumnas aprendan la ortografía en forma efectiva y la empleen, en la misma medida, las alumnas se habitúan a la autocorrección; que infieran las reglas ortográficas a partir de los textos ortográficos leídos por ellas. Estos resultados coinciden con la investigación de Quiñones (2010) quien aplicando un método de lectura comprobó que el $100 \%$ de los alumnos mejoraron sus problemas de mala ortografía, después de leer textos especialmente seleccionados.

Palabras clave: Creación, lectura, ortografía, técnica Lecretexor, textos.

\begin{abstract}
The principal objective of investigation serves to be the determination of the form in than the application of the technique LECRETEXOR (reading and creation of orthographic texts) influences the learning of orthography. The methodology used was the cuasiexperimental. The subjects of the cross - section constituted the schoolgirls of the first grade of secondary (First A, Experimental group) and (First B, Control group) of private school of misses Juan Landázuri of Cusco. The principal conclusion of investigation are: Than applying the technique LECRETEXOR in several learning sessions, it turns out well that schoolgirls learn the orthography in effective form and use her, in the same measure, the schoolgirls become addicted to the autocorrection; That they infer the orthographic rules as from the orthographic texts read by them. These results coincide with the research of Quiñones (2010) who, applying a reading method, found that $100 \%$ of the students improved their bad spelling problems, after reading specially selected texts.
\end{abstract}

Keywords: Creation, reading, orthography, technique Lecretexor, texts.

\section{Introducción}

Los hermanos Miguel y Manuel Inga Arias, refieren una anécdota que a continuación transcribimos: “Albert Einstein estudió en una escuela donde lo obligaban a memorizar los textos sin ningún tipo de reflexión y, por lo

571

Downloadable from: http://revistas.unu.edu.pe

Carretera Federico Basadre Km 6, Dirección de Producción Intelectual

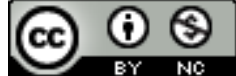

Revista de Investigación Universitaria por Universidad Nacional de Ucayali se distribuye bajo una Licencia Creative Commons Atribución-NoComercial 4.0 Internacional. 
tanto, no le permitían dialogar en clase con sus compañeros ni mucho menos hacer preguntas al maestro..." (2008, p. 56).

La enseñanza de la ortografía se ha parecido y se parece mucho a la enseñanza practicada con Einstein: no ha sido si no la memorización mecánica de reglas y más reglas, con sus respectivos ejemplos y muchas excepciones que terminan confundiendo al estudiante $\mathrm{y}$, peor aún, mentalizando en el alumno la idea que la ortografía, con su serie de reglas y excepciones, es aburrida.

La forma recurrente de la enseñanza de la ortografía que en la actualidad practican en Cusco los docentes de Gramática, consiste en dictar reglas y más reglas ortográficas con sus respectivos ejemplos y excepciones, forzando, con esta forma de enseñanza, solo la memorización y no el razonamiento y la aplicación consciente de las reglas ortográficas mediante la lectura y escritura. Esta forma de enseñanza ha hecho mucho daño a los estudiantes, por su carácter anticientífico y mecánico. Pues, los discentes conocen y repiten automáticamente dichas reglas, pero al momento de la escritura no las aplican, porque solo memorizaron y no asimilaron de manera razonable o consiente.
Este es el problema ortográfico también de las señoritas alumnas del Primer Grado de Secundaria de la Institución Educativa Particular de Señoritas "Juan Landázuri Ricketts" - Cusco, quienes presentan una serie de errores ortográficos en la escritura de palabras, de las mayúsculas, en la tildación y el mal empleo de los signos de puntuación. Hecho que se constató notoriamente la revisión de los cuadernos y otros textos escritos, evaluaciones escritas, ejercicios en la pizarra y revisión de las asignaciones o monografías. Y, principalmente, con la aplicación del Pre test en alumnas de primer grado de secundaria.

En el inconsciente de la mayoría de las personas, la ortografía es un castigo que implica escribir 50 o 100 veces la palabra errada, además, que es aburrida, por lo mismo, genera malestar o da miedo. Frente a ello, en esta tesis y la técnica mencionada se propone, convertir la ortografía en una actividad agradable e interesante, hasta que en el cerebro de las alumnas se active (a nivel consciente y subconsciente) esa impresión y sensación de agrado e interesante que tiene la ortografía. De tal manera que, en adelante, siempre que la alumna entre en contacto con la escritura, se activará automáticamente en el subconsciente la sensación e impresión mencionadas. 
Por otra parte, el aprendizaje y práctica de la ortografía de una lengua no se logra conociendo las reglas solamente, sino, con una lectura atenta y fijativa y la práctica constante de la escritura, la escucha de las palabras correctamente pronunciadas y la corrección habitual de lo escrito. Es decir, se aprende a escribir bien, leyendo, escribiendo, escuchando y corrigiendo lo escrito.

Para la Real Academia Española, la ortografía es un conjunto de normas que regulan la correcta escritura de una lengua y una disciplina lingüística. La RAE dice:

"El término ortografía designa asimismo la disciplina lingüística de carácter aplicado que se ocupa de describir y explicar cuáles son los elementos constitutivos de la escritura de una lengua y las convenciones normativas de su uso en cada caso, así como los principios y criterios guían tanto la fijación de las reglas como sus modificaciones. (2010, págs. 9 -11).

Como todo aprendizaje, el de la escritura y la práctica de esta, es una actividad neurolingüística; es decir, tiene su base dentro del cerebro. Los neurolingüistas han determinado que el lenguaje escrito tiene una zona en el cerebro llamada área de Exner. Esta se sitúa en el hemisferio cerebral izquierdo, más arriba de la zona de Broca (zona del lenguaje hablado). De tal forma que, si un ser humano no puede escribir pese a todos los estímulos para que lo haga es porque está dañada la zona Exner de su cerebro y requiere la intervención y tratamiento de un neurólogo. Pero, específicamente la mala escritura, no es un problema neurolingüístico. Es decir, no se escribe con errores ortográficos a causa de un daño cerebral. Es una cosa no poder escribir y otra muy distinta, no querer escribir bien o escribir con errores ortográficos o no querer corregir lo que se escribe. La mala ortografía es, más bien, un problema socioeducativo causado por falta de una conciencia y hábito por escribir bien de las mismas personas o por falta de una enseñanza significativa de parte del docente. En ese sentido, no son los neurólogos quienes han de mejorar la escritura correcta de las palabras, sino, el mismo alumno, claro está, con la guía del docente.

La palabra función se asocia con la frase interrogativa para qué. En ese sentido, ¿para qué existe o para qué sirve la ortografía? La prestigiosa Real Academia Española (2010, p. 17), responde clara y contundentemente: "La función esencial de la ortografía es garantizar y facilitar la comunicación escrita entre los usuarios de una lengua mediante el 
establecimiento de un código común para su representación gráfica”.

RAE (2010, p. 23), explica:

En las lenguas de escritura alfabética, basadas en la utilización de signos gráficos para representar las unidades fónicas de la cadena hablada, la ortografía cumple, además, un papel esencial como factor de unidad... Así pues, la ortografía contribuye decisivamente a evitar la dispersión en la representación gráfica de una misma lengua, dispersión que, llevada al extremo, haría difícil y hasta imposible la comunicación escrita entre sus hablantes y comprometería su identificación como miembros de una sola comunidad lingüística...

Los hablantes alfabetizados ven en la lengua escrita el modelo de corrección y tiende a pronunciar las palabras de acuerdo con su forma gráfica...

Como se ha dicho y demostrado textos atrás, la lectura y la escritura se vinculan estrechamente. El lector capta, fija en su mente las letras de la palabra, infiere y generaliza ciertas reglas, detecta errores ortográficos en el texto que lee y corrige dicho empleo impropio. Seguro de ello, el maestro Albalat, recuerda esta gran verdad:

Todos los grandes escritores proclaman la necesidad de leer y de leer bien. La lectura es la base del arte de escribir... la lectura disipa la aridez, activa las facultades, la inteligencia y pone en libertad a la imaginación... la lectura es el gran secreto. Lo enseña todo, desde la ortografía hasta la construcción de las frases. (2008, págs. 23 - 25).

Valladares consolida dicha opinión manifestando que:

La lectura permite tener buena ortografía. ¿Qué significa tener buena ortografía? Significa escribir las palabras con corrección y utilizar adecuadamente los signos de la escritura.

La ortografía, básicamente, se adquiere leyendo y escribiendo con frecuencia.

$\mathrm{Al}$ leer, memorizamos visualmente la forma cómo están escritas las palabras; y cuando escribimos, esas formas se manifiestan tal como fueron memorizadas. Por eso, los que tienen la costumbre de leer, por lo general, escriben sin errores ortográficos. (2007, p. 16). 
Los autores mencionados y sus respectivos textos no hacen más que consolidar la incuestionable y efectiva forma de aprender la ortografía a través de la lectura. En efecto los que leen siempre escriben bien, pues resulta que cuando se lee, la memoria visual capta no solo la palabra sino las letras que constituyen dicha palabra. La captación incluso es inconsciente, pero efectiva. Por esta razón, los que leen captan las grafías de los vocablos y escriben correctamente. Por ello, también la importancia de que lean textos ortográfica y textualmente bien redactados.
El diseño de la investigación está establecido en dos etapas:

La primera es el descriptivo - causal bivariado, cuyo esquema es el siguiente:

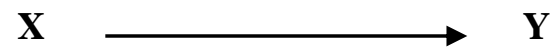

En el que $\mathbf{X}$ es variable independiente (aplicación de la técnica Lecretexor) que influye en $\mathbf{Y}$ variable dependiente (aprendizaje de la ortografía).

La segunda es el cuasiexperimental para la parte aplicativa. Dicho diseño se aplicó a dos grupos en la siguiente forma:

\section{Metodología}

\begin{tabular}{lcc}
\multicolumn{1}{c}{ ACTIVIDADES } & $\begin{array}{c}\text { GRUPO } \\
\text { EXPERIMENTAL }\end{array}$ & $\begin{array}{c}\text { GRUPO } \\
\text { CONTROL }\end{array}$ \\
\hline$\checkmark$ PRE-TEST: mejoramiento de la ortografía. & Y1 & Y2 \\
$\checkmark$ APLICACIÓN: lectura y creación de textos & Sí & No \\
$\begin{array}{l}\text { ortográficos. } \\
\checkmark \text { POST - TEST: mejoramiento de la ortografía. }\end{array}$ & Y3 & Y4 \\
\hline
\end{tabular}

Como se ha dicho, LECRETEXTOR es la abreviación de lectura y creación de textos ortográficos (redacción de relatos, anécdotas, poemas, crucigramas y otros). La aplicación de esta técnica es lúdica y primordialmente se realiza en la clase y comprende las actividades lectoras y de redacción creativa, teniendo en cuenta los modelos, técnicas, estrategias, decálogos, consejos y actividades sugeridas por los autores y citadas páginas más arriba. A continuación, se explican con mayor detalle.

Lectura. En esta fase, los alumnos:

- Leen textos ortográficos con el fin de tomar conciencia sobre la importancia de la ortografía y motivarse para el aprendizaje del tema a tratar.

- Leen textos ortográficos para que los tomen como modelo en la creación y, 
principalmente, para que se inspiren o se iluminen.

- Estudian reglas ortográficas para su conocimiento y análisis respectivo.

- Leen textos con palabras de escritura dudosa, fijándose intensamente hasta almacenarlas en su cerebro.

Creación. En esta fase, los alumnos con orientación del docente:

- Copian o transcriben textos con palabras de escritura dudosa, que han leído fijándose intensamente hasta almacenarlas en su cerebro.

- Redactan relatos ortográficos de manera creativa, imaginativa, original y humorística convirtiendo las reglas ortográficas en textos.

- Reescriben los relatos ortográficos leídos, mediante el dictado o la copia, con el fin de que las ideas se graben en el cerebro de los alumnos.

- Socializan o comparten entre ellos, los textos ortográficos creados.

- Confrontan los textos creados y leídos con las reglas ortográficas.

- Detectan los errores ortográficos en el texto leído.
- Perfeccionan o corrigen los errores ortográficos en el texto leído, consultando el diccionario y libros actualizados de ortografía.

- Reflexionan, mediante el diálogo, sobre el tema estudiado en la sesión y los procedimientos realizados.

\section{Resultados y discusión}

Nuestros resultados muestran que la media del puntaje Escritura y resolución de los ejercicios ortográficos en el Pretest del grupo experimental es $4.21 \pm 2.21$ y la media del puntaje Escritura y resolución de los ejercicios ortográficos en el Postest del Grupo Experimental es de 11.1 \pm 2.23 . Se observa que existe diferencias significativas $\mathrm{P}<0.05$, apreciándose mayor media en el Postest; es decir que, Aplicando la técnica LECRETEXOR en varias sesiones de aprendizaje, se logra que las alumnas escriban textos y resuelvan los ejercicios ortográficos de manera correcta. Es decir, las alumnas mejoran significativamente en el empleo adecuado de los signos de puntuación, en la correcta colocación de las tildes, el uso de las mayúsculas, y escritura de letras, palabras y textos. 


\section{Tabla 1}

Comparación de medias del puntaje Escritura y resolución de los ejercicios ortográficos en el Pretest y Postest del grupo experimental

\begin{tabular}{lccccc}
\hline $\begin{array}{c}\text { Escritura y resolución de los } \\
\text { ejercicios ortográficos }\end{array}$ & Media & N & $\begin{array}{c}\text { Desviación } \\
\text { estándar }\end{array}$ & T & P \\
\hline Pretest & 4,21 & 48 & 2,21 & 14,81 & $0.000^{*}$ \\
Postest & 11,10 & 48 & 2,13 & &
\end{tabular}

$* \mathrm{P}<0.05$ existe diferencias significativas

De la tabla se observa que la media del puntaje Escritura y resolución de los ejercicios ortográficos en el Grupo Experimental es $11.1 \pm 2.23$ y la media del puntaje Escritura y resolución de los ejercicios ortográficos en el Grupo Control es de 5.19 \pm 3.04 . Se observa que existe diferencias significativas $\mathrm{P}<0.05$, apreciándose que mayor media presenta el Grupo Experimental; es decir que, aplicando la técnica LECRETEXOR en varias sesiones de aprendizaje, se logra que las alumnas del Grupo Experimental resuelvan los ejercicios ortográficos de manera correcta y escriban textos sin errores ortográficos. Estos resultados coinciden con la investigación de Quiñones (2010) quien aplicando un método de lectura comprobó que el $100 \%$ de los alumnos mejoraron sus problemas de mala ortografía, después de leer textos especialmente seleccionados. Asimismo, demuestra que la aplicación de la lectura en el aprendizaje de la ortografía cumple un papel trascendental porque permitió que los alumnos al leer los textos se fijen en la correcta escritura de las palabras, y la lectura permite que los alumnos mentalicen la correcta forma gráfica de las palabras.

Tabla 2

Comparación de medias del puntaje Conciencia ortográfica en el Pretest y Postest del Grupo Experimental

\begin{tabular}{lccccc}
\multicolumn{1}{c}{ Conciencia ortográfica } & Media & N & $\begin{array}{c}\text { Desviación } \\
\text { estándar }\end{array}$ & T & P \\
\hline Pretest & 3,92 & 48 & 0,54 & 13.55 & $0.000^{*}$ \\
Postest & 6,90 & 48 & 1,40 & & \\
\hline
\end{tabular}

$* \mathrm{P}<0.05$ existe diferencias significativas.

De la tabla se observa que la media del puntaje

Conciencia ortográfica en el Pretest del
Grupo Experimental es $3.92 \pm 0.54$ y la media del puntaje Conciencia ortográfica en el 
Postest del Grupo Experimental es de 6.9 \pm 1.4. Se observa que existe diferencias significativas $\mathrm{P}<0.05$, apreciándose mayor media en el Postest, es decir que, Aplicando la técnica LECRETEXOR en varias sesiones de aprendizaje, se logra que las alumnas tomen conciencia sobre la importancia de la ortografía, en el campo de la comunicación escrita.

De la tabla se observa que la media del puntaje Conciencia ortográfica en el del Grupo Experimental es $6.9 \pm 1.4$ y la media del puntaje conciencia ortográfica en el Grupo Control es de 3.79 \pm 0.77 . Se observa que existe diferencias significativas $\mathrm{P}<0.05$; es decir que, Aplicando la técnica LECRETEXOR en varias sesiones de aprendizaje, se logra que las alumnas del Grupo Experimental tomen conciencia sobre la importancia de la ortografía, en el campo de la comunicación escrita. Coincide con la investigación de Choquehuanca (2006); La técnica del dictado en el aprendizaje de la ortografía en los alumnos del $3^{\circ}$ grado de secundaria Antes de la aplicación de la técnica del dictado el 99\% de los alumnos $3^{\circ}$ grado de secundaria del Colegio Estatal Mixto Jerónimo Zavala de Combapata- Canchis, tenían problemas de mala ortografía, incluso desconocían las reglas ortográficas. Aplicando la técnica del dictado los alumnos mejoraron sus problemas ortográficos en un $100 \%$, demostrándose que la técnica del dictado es efectiva para la enseñanza de la ortografía.

Tabla 3

Comparación de medias del puntaje Hábito de autocorrección ortográfica en el Pretest y Postest del Grupo Experimental

\begin{tabular}{lccccc}
\hline $\begin{array}{c}\text { Hábito de autocorrección } \\
\text { ortográfico }\end{array}$ & Media & N & $\begin{array}{c}\text { Desviación } \\
\text { típ. }\end{array}$ & T & P \\
\hline Pretest & 0,75 & 48 & 1,10 & 13.83 & $0.000^{*}$ \\
Postest & 2,17 & 48 & 0,83 & & \\
\hline
\end{tabular}

$* \mathrm{P}<0.05$ existe diferencias significativas

De la tabla se observa que la media del puntaje

Hábito de autocorrección ortográfico en el

Pretest del Grupo Experimental es 0.75 2.17

y la media del puntaje Hábito de autocorrección ortográfico en el Postest del

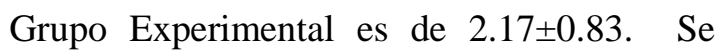

observa que existe diferencias significativas $\mathrm{P}<0.05$, apreciándose mayor media en el Postest; es decir que, al crear relatos ortográficos, identificando y corrigiendo sus errores permanentemente, las alumnas se habitúan a la autocorrección. 
De la tabla se observa que la media del puntaje hábito de autocorrección ortográfico en el Grupo Experimental es $2.17 \pm 2.17$ y la media del puntaje hábito de Autocorrección ortográfico en el Grupo Control es de $0.75 \pm 1.1$. Se observa que existe diferencias significativas $\mathrm{P}<0.05$, apreciándose mayor media en el Grupo Experimental; es decir que, al crear relatos ortográficos, identificando y corrigiendo sus errores permanentemente, las alumnas del Grupo Experimental lograron habituarse a la autocorrección ortográfica.

El aprendizaje de los aspectos más arbitrarios de las reglas gramaticales es la dificultad de memorizar reglas, recurso único a lo largo de mucho tiempo en la enseñanza tradicional: memorizar reglas, aplicarlas para llenar espacios en blanco y hacer dictados. Gómez Palacios (1998) afirma que basarse en estrategias didácticas para la enseñanza ortográfica tales como la memorización de reglas o la escritura repetida de palabras, no es suficiente para descubrir las normas ortográficas, porque no toda la ortografía puede resolverse recurriendo a reglas como se ha argumentado. Existen palabras que son determinadas por cuestiones históricas o porque los errores ortográficos no son producto de un descuido o del desconocimiento de las reglas, sino se aplican criterios particulares, como ya se mencionó en apartados anteriores, que las alumnas desarrollan y ponen a prueba.

\section{Tabla 4}

Comparación de medias del puntaje Inferencia de las reglas ortográficas en el Pretest y Postest del Grupo Experimental

\begin{tabular}{lccccc}
\hline $\begin{array}{c}\text { Escritura y resolución de los } \\
\text { ejercicios ortográficos }\end{array}$ & Media & N & $\begin{array}{c}\text { Desviación } \\
\text { típ. }\end{array}$ & T & P \\
\hline Pretest & 0.75 & 48 & 1.10 & 14,81 & $0.000^{*}$ \\
Postest & 2.17 & 48 & 0.83 & & \\
\hline
\end{tabular}

*P $<0.05$ existe diferencias significativas

De la tabla se observa que la media del puntaje Inferencia de las reglas ortográficas en el Pretest del Grupo Experimental es $0.75 \pm 1.1$ y la media del puntaje Inferencia de las reglas ortográficas en el Postest del Grupo Experimental es de $2.17 \pm 0.83$. Se observa que existe diferencias significativas $\mathrm{P}<0.05$, apreciándose mayor media en el Postest; es decir que, aplicando la técnica LECRETEXOR en varias sesiones de aprendizaje, se logra que las alumnas hagan Inferencia de las reglas ortográficas a partir de 
los textos ortográficos leídos. De la tabla se observa que la media del puntaje Inferencia de las reglas ortográficas en el Grupo Experimental es $2.58 \pm 0.61$ y la media del puntaje Inferencia de las reglas ortográficas en el Grupo Control es de 1.13 \pm 0.33 . Se observa que existe diferencias significativas $\mathrm{P}<0.05$, apreciándose mayor media en el Grupo Experimental; es decir que, aplicando la técnica LECRETEXOR en varias sesiones de aprendizaje, se logra que las alumnas logren buenas inferencias de las reglas ortográficas, después de leer textos ortográficos. El docente de Gramática, al encarar el fenómeno ortográfico, se encuentra con varios obstáculos, entre los que se encuentran el lugar que ocupa la ortografía dentro del currículum, el tiempo necesario para realizar la corrección de los errores y la imagen de materia carente de colorido e importancia que tienen los alumnos sobre la ortografía

Tabla 5

Comparación de medias del puntaje Estilos de aprendizaje en el Pretest y Postest del Grupo Experimental

\begin{tabular}{|c|c|c|c|c|c|}
\hline \multirow{2}{*}{$\begin{array}{c}\text { Inferencia de las reglas } \\
\text { ortográficas }\end{array}$} & \multicolumn{5}{|c|}{ Desviación } \\
\hline & Media & $\mathbf{N}$ & típ. & $\mathbf{T}$ & $\mathbf{P}$ \\
\hline Pretest & 8,88 & 48 & 3,06 & 14,81 & $0.000 *$ \\
\hline Postest & 20,17 & 48 & 2,75 & & \\
\hline
\end{tabular}

$* \mathrm{P}<0.05$ existe diferencias significativas.

De la tabla se observa que la media del puntaje Estilos de aprendizaje en el Pretest del Grupo Experimental es $8.88 \pm 3.06$ y la media del puntaje Estilos de aprendizaje en el Postest del Grupo Experimental es de 20.17 \pm 2.75 . Se observa que existe diferencias significativas $\mathrm{P}<0.05$, apreciándose mayor media en el Postest; es decir que, la aplicación de la técnica LECRETEXOR influye de manera efectiva en el aprendizaje de la ortografía de las alumnas del Primer Grado de Secundaria de la Institución Educativa Particular de Señoritas “Juan Landázuri Ricketts" - Cusco.
Los problemas ortográficos son de diversa índole y están debidamente clasificados por los distintos autores. De ahí que es importante prestar atención a aquellos aspectos que pueden afectar el aprendizaje ortográfico de la lengua, como es el caso de la ausencia de motivación para la ortografía. Para llegar a una verdadera aceptación y atención hacia este aspecto, el alumno tiene que ir hacia ella como fuera a una experiencia agradable y enriquecedora, y este aprendizaje hay que enseñarlo a través de una técnica lúdica, agradable, haciéndole ver que la ortografía es 
UNIVERSIDAD NACIONAL DE UCAYALI

Revista de Investigación Universitaria

Versión electrónica 2664 - 8423

ARTICULO ORIGINAL
Vol. $11 \mathrm{~N}^{\circ}$ 2, pp. 571 - 582, julio/diciembre 2021

Recibido18/07/2021

Aceptado 12/11/2021

Publicado 30/12/2021 importante dentro de la comunicación escrita, hasta que tome conciencia de esa importancia.

\section{Conclusiones}

Aplicando la técnica LECRETEXOR en varias sesiones de aprendizaje, se logra que las alumnas aprendan la ortografía en forma efectiva y la empleen, en la misma medida, las reglas ortográficas al redactar cualquier tipo de textos.

Aplicando la técnica LECRETEXOR en varias sesiones de aprendizaje, se logra, en forma significativa, que las alumnas tomen conciencia sobre la importancia de la ortografía en el campo de la comunicación escrita, y que juzguen de manera positiva a la ortografía, asumiendo conscientemente que, el aprendizaje de la ortografía no es castigo ni molestia; sino, fácil, agradable, interesante, útil y significativo.

Al crear textos ortográficos, identificando y corrigiendo sus errores permanentemente, las alumnas se habitúan a la autocorrección. Entendiendo que corregirse es perfeccionarse. Aplicando la técnica LECRETEXOR las alumnas mejoran en forma fácil y significativa su Inferencia de las reglas ortográficas a partir de los textos ortográficos leídos por ellas.

Aplicando la técnica LECRETEXOR las alumnas logran en forma significativa la
Creación de textos ortográficos con originalidad y sin error.

\section{Referencias bibliográficas}

Albalat, A. (2008). El arte de escribir y la formación del estilo. Edición Ministerio de Cultura.

Arribasplata, Miguel y otros. (2010). Usos y Abusos del Idioma. Editorial San Marcos.

Choquehuanca (2006). Influencia de la lectura en la enseñanza de la ortografía en los alumnos del 3er de secundaria del Colegio Estatal Mixto Jerónimo Zavala de Combapata - Canchis. [Tesis para optar el título profesional de licenciado en educación, especialidad de Lengua y Literatura en la Universidad Nacional de San Antonio Abad del Cusco].

Gómez, M. (1998). Talleres de escritura. México. Editorial SEEP Pronalees.

Inga Arias, Miguel y Manuel Inga A. (2008).

Desarrollo de las habilidades comunicativas. Centro de Producción Fondo Editorial - UNMSM.

López Sánchez, Pepe. (2006). Cuentos ortográficos. Con un poco de humor. Edita: M.R.P. AULA LIBRE. 
UNIVERSIDAD NACIONAL DE UCAYALI

Revista de Investigación Universitaria

Versión electrónica 2664 - 8423

ARTICULO ORIGINAL
Vol. $11 N^{\circ} 2$, pp. 571 - 582, julio/diciembre 2021

Recibido18/07/2021

Aceptado 12/11/2021

Publicado 30/12/2021
Palomino, N. (2015). Aplicación de la técnica lecretexor para el aprendizaje efectivo de la ortografía de las alumnas de la I. E. Juan Landázuri Ricketts de Cusco, 2012. [Tesis para optar el grado académico de magister en educación, mención didáctica de la comunicación en EBR en la EPG de la Universidad Nacional Mayor de San Marcos].

Quiñones (2010). La enseñanza de la ortografía a través de la lectura de los estudiantes del 4to de secundaria de la Gran Unidad Escolar Inca Garcilaso de la Vega de Cusco. [Tesis para optar el título profesional de licenciado en educación, especialidad de Lengua y Literatura en la Universidad Nacional de San Antonio Abad del Cusco]

Real Academia Española y Asociación de Academias de la Lengua Española: (2005) Diccionario panhispánico de dudas. Edit. Aguilar. (2010) Nueva gramática de la lengua española. Editorial ESPASA CALPE, S. A. (2010) Ortografía de la lengua española. Editorial ESPASA CALPE, S. A.
(2013) Ortografía escolar de la lengua española. Edit. ESPASA LIBROS, S. L. U.

Valladares, O. (2007). Manual de ortografía. S.e. 